\title{
Progresses in the validation of the FLUKA atmospheric $\nu$ flux calculation
}

\author{
G. Battistoni ${ }^{\mathrm{a}}$, A. Ferrari ${ }^{\mathrm{b}}$, T. Montaruli ${ }^{\mathrm{c}}$ and P.R. Sala ${ }^{\mathrm{b}}$

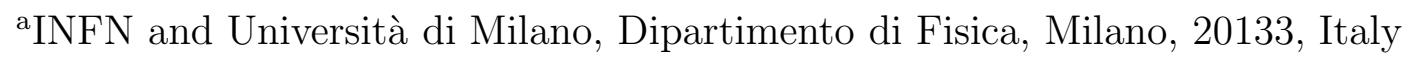 \\ ${ }^{\mathrm{b}}$ CERN, Geneva 23, Switzerland \\ ${ }^{\mathrm{c} I N F N}$ and Università di Bari, Dipartimento di Fisica, Bari, 70126, Italy
}

The FLUKA calculation of the atmospheric neutrino fluxes have been cross-checked by comparing predictions on lepton fluxes in atmosphere to experimental data. The dependence of predicted neutrino fluxes on the shape and normalization of primary spectrum is also investigated.

\section{Introduction}

The two essential features of the atmospheric neutrino fluxes, namely the up-down symmetry and the $\mu / e$ ratio, are now considered to be largely independent from the calculation scheme[目]. Theoretical uncertainties should still have an impact, together with experimental systematics, in the determination of the relevant physical parameters of neutrino oscillations 2]. However, the Super-Kamiokande allowed region in the $\Delta m^{2}-$ $\sin ^{2}(2 \Theta)$ space remains more or less invariant [3] when the Monte Carlo predictions change from the original HKKM results [4] to the FLUKA ones [5], despite the fact that the two set of predictions have important differences. The most significant one is the normalization of the flux, which, for the same neutrino cross-sections, gives a $15 \div 20 \%$ lower event rate in the case of FLUKA. The same ratio approximately exists between FLUKA and the original Bartol flux [6] for the same primary flux. This difference is due to the features of the hadronic particle production model. Our claim is that FLUKA has already given a more accurate prediction than the previous calculations. Also in the recent work of the Bartol group [7], in which some feature of their hadronic interaction model have been corrected, a neutrino flux very close to our predictions is now obtained for high cutoff sites, like Super-Kamiokande. It is likely that in the case of Super-Kamiokande analysis the differences between fluxes are in practice obscured by the assumed systematic uncertainties. However, in view of possible future improvements, it is important to clarify the differences among the existing predictions. The effects due to the $3-\mathrm{D}$ treatment of the shower, first presented in ref. [8], have been reproduced by other groups [9, 10 and are by now accepted as the correct prediction. The uncertainties in the neutrino rates are instead less clear, but they can be attributed to three independent sources: i) the primary cosmic ray spectrum, ii) the hadronic interaction model and iii) the neutrino-Nucleus cross sections. The first goal of this paper (section 2) is to provide further evidence that the particle yields predicted by the FLUKA model[8,1] are in the right range. As a second 
step, we consider the dependence of the predicted $\nu$ flux on the choice of primary spectrum (section 3). The fundamental topic of neutrino cross-sections is instead postponed to a future work. In the conclusions we summarize the arguments, advancing some criticism about other new attempts of $\nu$ flux calculations.

\section{New benchmarks for the FLUKA predictions}

FLUKA is constructed upon a set of theoretically inspired models of hadronic interactions, adjusting parameters only on the basis of results coming from accelerators. Cosmic ray data can however be used to check their quality. Recently two remarkable results have been achieved in this field. The first one is the reproduction of the features of the primary proton flux as a function of geomagnetic latitude as measured by AMS 11], thus showing that both the production of secondary nucleons and the geomagnetic effects and the overall geometrical description of the 3-D setup are well under control. The same work also shows that the fluxes of secondary $e^{+}$and $e^{-}$measured at high altitude are reproduced, and this is instead more directly linked to the yield of mesons produced in primary interactions. The second achievement is the good reproduction of the muon data in atmosphere as measured by the CAPRICE experiment [12, both at ground level and at different floating altitudes [13], when starting from the same primary flux (Bartol fit) [6] used to generate atmospheric neutrinos. The fluxes of atmospheric muons are strictly related to the neutrino ones, because almost all $\nu$ 's are produced either in association, with, or in the decay of $\mu^{ \pm}$. The level of agreement reported in ref.[13], without any adjustment of the model, allows to conclude that, the normalization of the $\nu$ fluxes predicted by FLUKA is in the right range. This is true for a choice of primary spectrum constrained by the more recent data, in particular from AMS [14] and BESS 15. The quoted primary flux of ref.[6] is already in good, although not yet optimal, agreement with these data. Furthermore, the agreement exhibited by the FLUKA simulation separately for muons of both charges gives confidence on the simulated yields of $\pi^{+}$and $\pi^{-}$production in air. This is one of the main sources of uncertainty in the prediction of the $\mu / e$ ratio. The decay chain of $\pi^{+}$eventually results in $\nu_{e}$, while the decay of $\pi^{-}$generates $\bar{\nu}_{e}$. Due to the different interaction cross section this affects the experimental event rates.

\section{Neutrino Fluxes and Primary Spectrum}

For a given shower model, the level of agreement in a comparison to measured particle fluxes in atmosphere depends on the parameterization of the primary spectrum. The primary choice originally used in ref. [- [t] would force in FLUKA a too high normalization of the flux, since it is includes the data points taken from the compilation of Webber et al. [16], now excluded by recent experiments. On the contrary, a constraint based on the primary data from CAPRICE[17 would not allow in FLUKA a satisfactory level of agreement with muon fluxes. As already mentioned, the Bartol fit used so far is not completely satisfactory. The scientific community is trying to establish a common reference for the primary flux to be used in all computations. An attempt in this direction has been proposed in ref.[18], where the statistical errors of measurements and the systematic differences between experiments are used to establish a band of uncertainty around the average result of this new fit. Contributions heavier than primary protons are also con- 
sidered. A similar attempt has been proposed in ref[10]. We have used the new Bartol fit of primaries to recalculate the FLUKA $\nu$ fluxes. As an example, in Fig. 1 1 we plot the ratio of the computed $\nu_{\mu}$ flux for the site of Super-Kamiokande as resulting from the new Bartol fit with respect to the old one, as a function of $\nu$ energy.

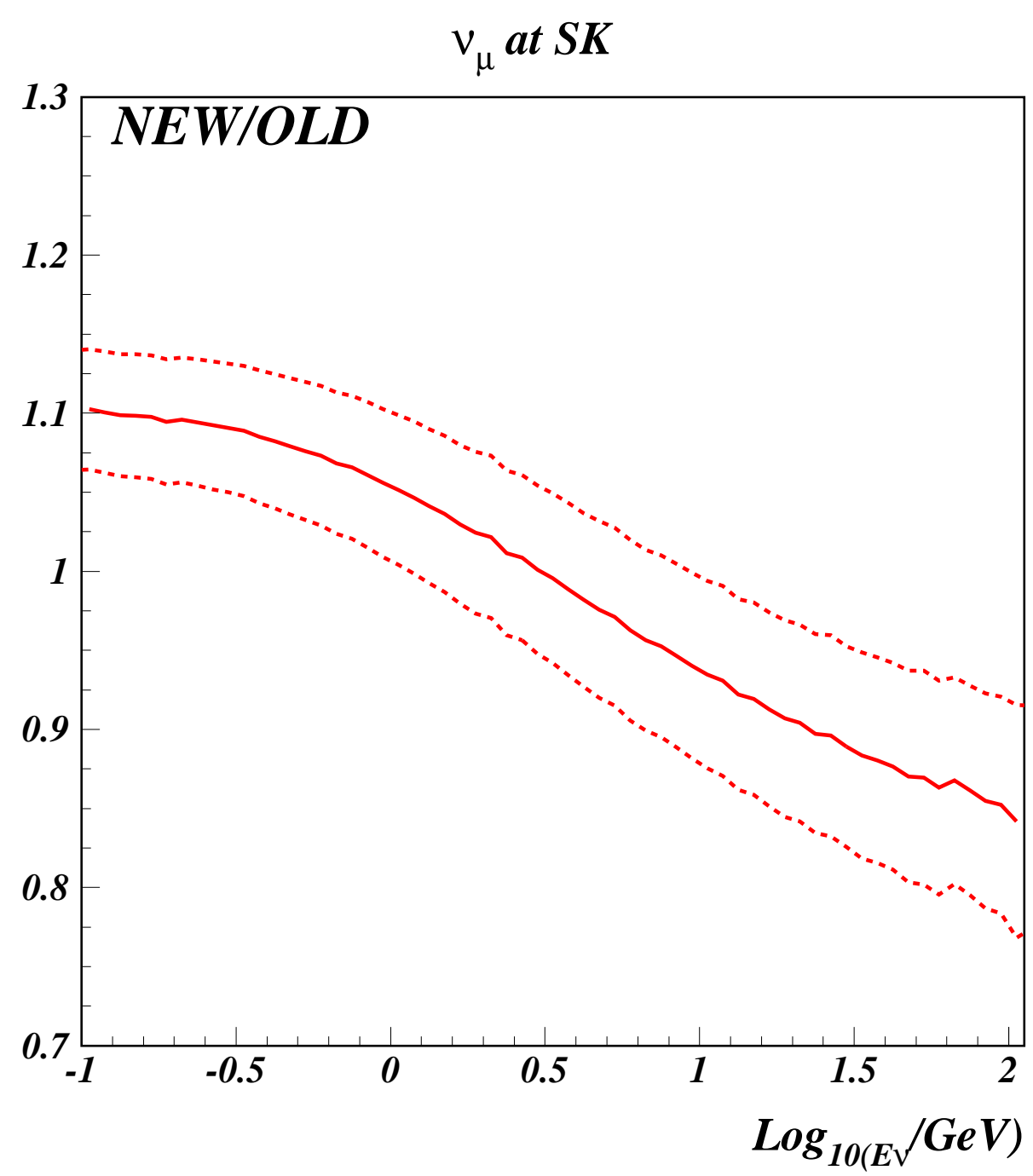

Figure 1. Ratio of the computed $\nu_{\mu}$ flux at Super-Kamiokande as resulting from the new Bartol fit with respect to the old fit, as a function of $\nu$ energy. The uncertainty band is also shown.

The new parameterization brings to an increase of neutrino fluxes in the Sub-GeV range, while a depletion occurs in the Multi-GeV range. This behavior is still maintained when event rates are considered. Integrating in energy, the average prediction for the atmospheric neutrino event rates increases, approximately for all flavors, by $\sim 5 \%$ with respect to the original evaluation, with an uncertainty (only due to the primary spectrum) of $\sim \pm 4 \%$. 


\section{Conclusions}

The absolute normalization of atmospheric neutrino fluxes is still a subject of investigation. The results summarized in this paper support the conclusion that the normalization of the FLUKA results should be close to reality and that the difference with respect to the original Bartol and HKKM results cannot be attributed to an insufficient particle production yield in the shower development in the FLUKA model. The knowledge of the primary spectrum is still an important issue, but the convergence towards a common reference, constrained by the AMS and BESS data allows to reduce the related uncertainty $( \pm \sim 5 \%)$. After this study we can also conclude that the agreement of FLUKA predictions to Super-Kamiokande[3] data will certainly improve if the updated parameterization of the proton and He fluxes are used. This should be more evident for the Sub-GeV events. This does not mean that the questions about the absolute values of neutrino cross-sections and the hadronic interaction descriptions are answered. In particular we remind that hadron-Nucleus and Nucleus-Nucleus interactions in the energy range $1 \div 30 \mathrm{GeV}$ are still insufficiently known to allow the construction of a fully reliable model. In any case it seems difficult that the overall uncertainties are larger than $15 \% \div 20 \%$. Therefore, in our opinion, the results on neutrino flux of ref. [19], starting from a primary spectrum very similar to the one here considered, seem too low in the Sub-GeV region, not being compatible with the corresponding muon fluxes in atmosphere, unless significant systematic error in the data at high altitude are assumed. Furthermore, after our tests on direct Nucleus-Nucleus interaction, by means of an interface to the DPMJET code[20], we cannot share their conclusion about the existence of a significant bias introduced by the use of the superposition model. As far as the $\nu$ fluxes proposed in ref. 221, we believe that they are probably affected by some severe error (see also ref.[2]). Beyond other considerations, those results would imply, without any supporting evidence, that either the experimental data on atmospheric neutrinos are strongly biased or that the present knowledge of neutrino cross sections are wrong by an unexplained large factor.

\section{REFERENCES}

1. G. Battistoni, hep-ph/0012268, and Nucl. Phys. Proc. Suppl. 100 (2001) 101.

2. P. Lipari, Proc. of the IX Workshop on Neutrino Telescopes, Venezia (Italy) 2001.

3. Y. Totsuka, these proceedings.

4. M. Honda et al., Phys. Rev. D52 (1995) 4985.

5. A. Ferrari et al., references and documentation availabel at http://www.cern.ch/fluka.

6. V. Agrawal et al., Phys. Rev. D53 (1996) 1314.

7. T.K. Gaisser, proc. of Taup2001, Sep 8-12 2001, Assergi (Italy); R. Engel, et al. Proc. of the 27th ICRC (Hamburg, 2001), Session HE2.02.

8. G. Battistoni et al., Astrop. Phys. 12 (2000) 315. FLUKA flux tables are available at http://www.mi.infn.it/ battist/neutrino.html.

9. P. Lipari, Astrop. Phys. 14 (2000) 153.

10. M. Honda et al., Proc. of the 27th ICRC (Hamburg, 2001), Session HE2.04.

11. P. Zuccon et al., proc. of Taup2001, Sep. 8-12 2001, Assergi, Italy. Also: astroph/0111111, to be submitted to Phys. Lett. B.

12. M. Boezio et al., Phys. Rev. D62 (2000) 032007. 
13. G. Battistoni et al., hep-ph/0197241, to be published in Astroparticle Phys.

14. J. Alcaraz et al., Phys. Lett. B472 (2000) 215.

15. T. Sanuki et al., Astrophys. J. 545 (2000) 1135.

16. W.R. Webber, R.L. Golden \& S.A. Stephens, Proc. 20th ICRC (Moscow) vol. 1 (1987) 325 .

17. M. Boezio et al., Astrophys. J. 518 (1999) 457.

18. T.K. Gaisser et al., Proc. of the 27th ICRC (Hamburg, 2001), Session OG1.01

19. G. Fiorentini, V.A. Naumov, F.L. Villante, hep-ph/0103322.

20. J. Ranft, hep-ph/9911213; S. Roesler et al., hep-ph/0012171.

21. V. Plyaskin, hep-ph/0103286 